\title{
Observation of Bogoliubov excitations in exciton-polariton condensates
}

\author{
S. UTSUNOMIYA ${ }^{1,2,3 *}$, L. TIAN ${ }^{4}$, G. ROUMPOS ${ }^{4}$, C. W. LAll ${ }^{1,4}$, N. KUMADA ${ }^{3}$, T. FUJISAWA ${ }^{3}$, \\ M. KUWATA-GONOKAMI ${ }^{5}$, A. LÖFFLER ${ }^{6}$, S. HÖFLING ${ }^{6}$, A. FORCHEL ${ }^{6}$ AND Y. YAMAMOTO ${ }^{1,2,4 *}$
}

\author{
${ }^{1}$ National Institute of Informatics, Hitotsubashi, Chiyoda-ku, Tokyo 101-8430, Japan \\ ${ }^{2}$ Department of Information and Communication Engineering, The University of Tokyo, Tokyo 113-8654, Japan \\ ${ }^{3} \mathrm{NTT}$ Basic Research Laboratories, NTT Corporation, Atsugi, Kanagawa 243-0198, Japan \\ ${ }^{4}$ E. L. Ginzton Laboratory, Stanford University, Stanford, California 94305-4088, USA \\ ${ }^{5}$ Department of Applied Physics, The University of Tokyo, 7-3-1 Hongo, Bunkyo-ku, Tokyo 113-8656, Japan \\ ${ }^{6}$ Technische Physik, Universität Würzburg, Am Hubland, D-97074 Würzburg, Germany \\ *e-mail: shoko@nii.ac.jp; yyamamoto@stanford.edu
}

Einstein's 1925 paper predicted the occurrence of Bose-Einstein condensation (BEC) in an ideal gas of non-interacting bosonic particles ${ }^{1}$. However, particle-particle interaction and peculiar excitation spectra are keys for understanding BEC and superfluidity physics. A quantum field-theoretical formulation for a weakly interacting Bose condensed system was developed by Bogoliubov in 1947, which predicted the phonon-like excitation spectrum $^{2}$ in the low-momentum regime. The experimental verification of the Bogoliubov theory on the quantitative level was carried out for atomic $\mathrm{BEC}^{3}$ using the two-photon Bragg scattering technique ${ }^{4}$. Exciton-polaritons in a semiconductor microcavity, which are elementary excitations created by strong coupling between quantum-well excitons and microcavity photons, were proposed as a new BEC candidate in solid-state systems ${ }^{5}$. Recent experiments with exciton-polaritons have demonstrated several interesting signatures from the viewpoint of polariton condensation, such as quantum degeneracy at non-equilibrium conditions $s^{6-8}$, the polariton-bunching effect at the condensation threshold ${ }^{9}$, long spatial coherence ${ }^{10-12}$ and quantum degeneracy at equilibrium conditions ${ }^{13}$. The particle-particle interaction and the Bogoliubov excitation spectrum are at the heart of BEC and superfluidity physics, but have only been studied theoretically for exciton-polaritons ${ }^{14,15}$. In this letter, we report the first observation of interaction effects on the exciton-polariton condensate and the excitation spectra, which are in quantitative agreement with the Bogoliubov theory.

In a semiconductor microcavity with single or multiple quantum wells (QWs), eigenstates are altered to the new normal modes, called exciton-polaritons, when the cavity photon/QW-exciton coupling rate exceeds the decay rates of the photon and exciton. The exciton-polariton is a promising solid-state system for studying the dynamical condensation phenomena in solids ${ }^{5,16}$. Because its effective mass is eight orders of magnitude smaller than that of a hydrogen atom and four orders of magnitude smaller than exciton mass, the critical temperature of the polariton Bose-Einstein condensation (BEC) transition is expected to be up to room temperature. The leakage photons carry identical energy and in-plane momentum to the internal polaritons, thus it is possible to directly measure the energy-momentum dispersion relation and population distribution of the polaritons by an angle-resolved spectroscopy technique. This important information is available for liquid- ${ }^{4} \mathrm{He}$ systems only through 'quantum evaporation'17 and for gaseous atoms only through 'Feshbach resonance controlled free expansion'.

The exciton-polariton trap used in our experiment is shown in Fig. 1a. Three stacks of four GaAs QWs are embedded at the central three antinode positions of an AlAs/AlGaAs distributed Bragg reflector planar microcavity. The normal-mode splitting is $2 g_{0} \sim 15 \mathrm{meV}$ and the cavity photon lifetime is $2 \mathrm{ps}$. This leads to a $k=0$ lower-polariton (LP) lifetime of $\sim 4$ ps at zero detuning, $\Delta \equiv E_{\mathrm{C}}-E_{X}=0$, where $k$ is the in-plane wavenumber and $E_{\mathrm{C}}$ and $E_{X}$ are the cavity and QW exciton energies at $k=0$. The trap potential of $\sim 200 \mu \mathrm{eV}$ is provided by a hole surrounded by a thin metal $(\mathrm{Ti} / \mathrm{Au})$ film ${ }^{18}$. The cavity resonant field normally has an antinode at the AlGaAs-air interface. However, the antinode position is shifted inside the AlGaAs layer with the metal film as shown in Fig. 1a, which results in a blueshift of the cavity resonance and the LP energy. In our experiment, the LPs were confined in circular holes of varying diameters from $5 \mu \mathrm{m}$ to $100 \mu \mathrm{m}$. In a trap with 5-10 $\mu \mathrm{m}$ diameter, a single fundamental transverse mode dominates the condensation dynamics over other higherorder modes owing to the relatively weak confining potential.

The total number $n$ of LPs injected into a trap by the pump pulse and the number $n_{0}$ of the LP centred at $k=0$ and within $|k| \leqslant 4 \times 10^{3} \mathrm{~cm}^{-1}$, corresponding approximately to the trapped ground state, are plotted in Fig. $1 \mathrm{~b}$ as a function of normalized pump rate $P / P_{\text {th }}$. The fractional ratio $n_{0} / n$ increases nonlinearly at the condensation threshold and reaches a maximum of $\sim 0.6$ at $P / P_{\text {th }} \sim 6$.

The top panels in Fig. 1c show the near-field emission patterns from a trap with $8 \mu \mathrm{m}$ diameter at pump rates below, just above and well above the condensation threshold. The measured standard deviations for the polariton position $\Delta x$ and the wavenumber $\Delta k$ are plotted as functions of $P / P_{\text {th }}$ in Fig. 1c. Sudden decreases in $\Delta x$ and $\Delta k$ were clearly observed at $P \cong P_{\text {th }}$. Just above threshold, the measured uncertainty product $\Delta x \Delta k$ is $\sim 0.98$, which may be compared to the Heisenberg limit $(\Delta x \Delta k \sim 0.5)$ for a minimum-uncertainty wavepacket. The monotonic increase 
a

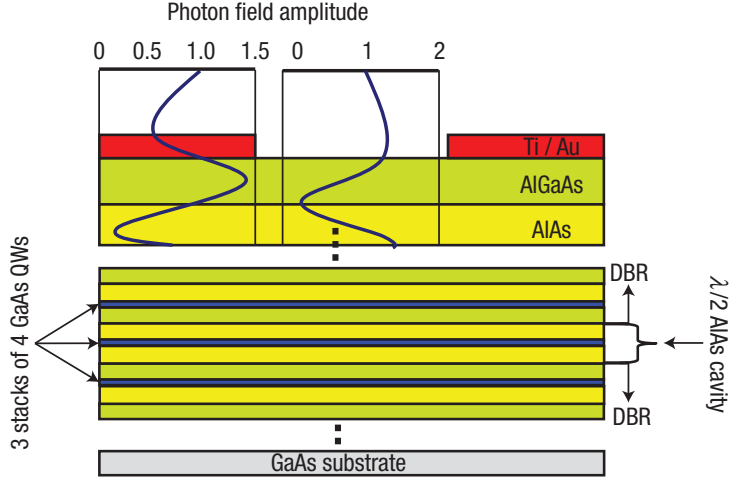

$\mathbf{G}$

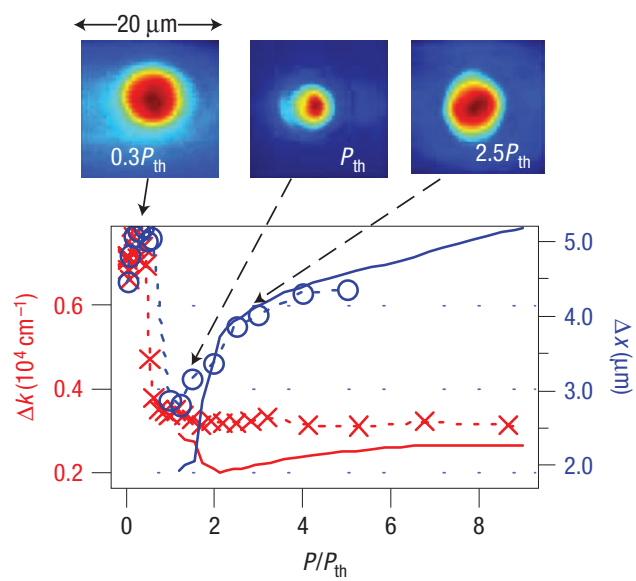

b

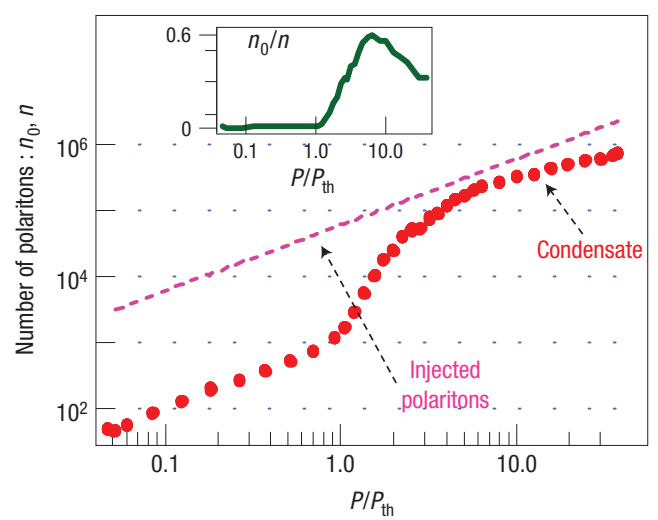

d

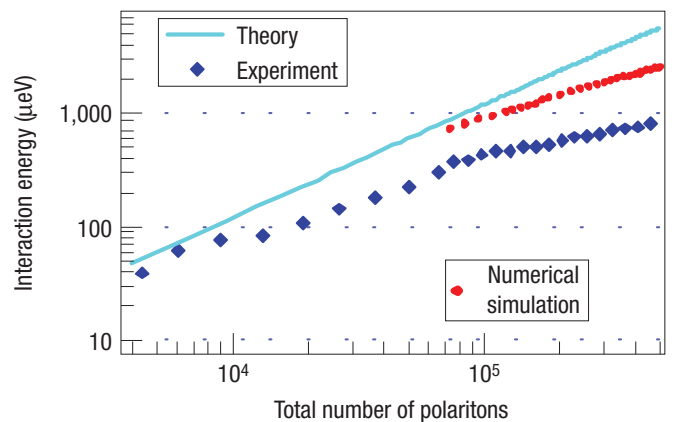

Figure 1 An exciton-polariton condensate in a single-mode trap. a, A schematic diagram of a polariton trap formed by a thin metal film (Ti/Au: $4 / 20 \mathrm{~nm}$ ) on top of a microcavity structure with circular holes (diameter : 4-100 $\mu \mathrm{m}$ ). The microcavity consists of a $\lambda / 2$ AlAs optical cavity layer sandwiched by two distributed Bragg reflectors with alternating AIGaAs/AIAs $\lambda / 4$ layers, where $\lambda$ is the cavity resonance wavelength. Three stacks of four GaAs QWs are placed at the central three antinodes of the microcavity photon field. A photon-field amplitude has an antinode at an AlGaAs-air interface without a metal film, whereas a deposited thin metal film pushes an antinode position inside the AIGaAs layer, which results in the blueshift of the cavity resonance and the lower polariton (LP) energy. The blueshift of the LP energy under the metal film is typically $\sim 200 \mu \mathrm{eV}$. b, The total number $n$ of LPs injected into a trap (pink dashed line) and number $n_{0}$ of the LP centred at $k=0$ and within $|k| \leqslant 4 \times 10^{3} \mathrm{~cm}^{-1}$ (red circles), which approximately corresponds to the LP number of the ground state of the trap, are plotted as functions of $P / P_{\text {th }}$. Inset: The fractional population $n_{0} / n$ as a function of $P / P_{\text {th }}$. The linearly polarized laser beam is injected with an angle of $60^{\circ}\left(k=7 \times 10^{4} \mathrm{~cm}^{-1}\right)$ into a trap with $8 \mu \mathrm{m}$ diameter, where the detuning parameter is $\Delta=5.4 \mathrm{meV}$. c. The measured standard deviations of LP distribution in coordinate $\Delta x$ (blue circles) and in wavenumber $\Delta k$ (red crosses) are plotted as a function of $P / P_{\mathrm{th}}$. Theoretical values for $\Delta x$ and $\Delta k$ obtained by the GP equation are shown by blue and red solid lines (Supplementary Information, S1). Top panels are the near-field images at three different pump levels: $0.3 P_{\text {th }}, P_{\text {th }}$ and $2.5 P_{\text {th }}$. d, The measured LP energy shift at $k=0$ (blue diamonds) and energy shift $U(n)$ calculated by equation (1) (light-blue solid line) are plotted as functions of the total number of polaritons. The numerical results by the GP equation, including the effect of pump-dependent condensate size, are shown by red circles (Supplementary Information, S1).

in $\Delta x$ and $\Delta x \Delta k$ at higher pump rates stems from the repulsive interaction among LPs in a condensate and is well reproduced by theoretical analysis using the Gross-Pitaevskii (GP) equation as shown in Fig. 1c (Supplementary Information, S1 for detailed theoretical analysis and Supplementary Information, S2 for complete experimental data.)

The $k=0$ LP energy is blueshifted with the number of polaritons as shown in Fig. 1d. This is a direct manifestation of the aforementioned repulsive interaction among LPs in a condensate. The $k=0$ LP energy shift $U(n)$ is calculated by the relation

$$
U(n)=\frac{1}{2} \delta E_{X}+\sqrt{g_{0}^{2}+\frac{\Delta^{2}}{4}}-\sqrt{g(n)^{2}+\frac{\left(\Delta-\delta E_{X}\right)^{2}}{4}},
$$

where $\delta E_{X}=E_{\mathrm{B}}\left(n / n_{\mathrm{s}}\right)$ and $g(n)=g_{0}\left(1-\left(n / n_{\mathrm{s}}^{\prime}\right)\right)$ represent the blueshift of the QW exciton energy due to fermionic exchange interaction ${ }^{19,20}$ and the reduced normal-mode splitting due to phase-space filling and fermionic exchange interaction $^{21}$, respectively. $n_{\mathrm{s}}=\left(N_{\mathrm{QW}} S / 2.2 \pi a_{\mathrm{B}}^{* 2}|X|^{2}\right)$ and $n_{\mathrm{s}}^{\prime}=\left(N_{\mathrm{QW}} S / 4 \pi a_{\mathrm{B}}^{* 2}|X|^{2}\right)$ are the saturation polariton numbers for the above two nonlinear processes, respectively. $a_{\mathrm{B}}^{*}$ is the QW exciton Bohr radius, $N_{\mathrm{QW}}=12$ is the number of QWs, $g_{0} \approx 7.5 \mathrm{meV}$ is the photon-exciton coupling strength, $S$ is the cross-sectional area of the condensate and $|X|^{2}=(1 / 2)\left[1+\Delta / \sqrt{4 g_{0}^{2}+\Delta}\right]$ is the exciton fraction of the $k=0 \mathrm{LP}$.

If we ignore the pump-rate dependency of the condensate cross-sectional area and assume a constant cross-sectional area determined by the trap area $S=\pi\left(4 \times 10^{-4} \mathrm{~cm}\right)^{2}$, the theoretical energy shift is shown by the light-blue line in Fig. 1d. We can numerically solve the GP equation to incorporate the pump-rate-dependent condensate size (see Supplementary Information, S1). The results are shown by red circles in Fig. 1d. These two theoretical predictions are compared with 

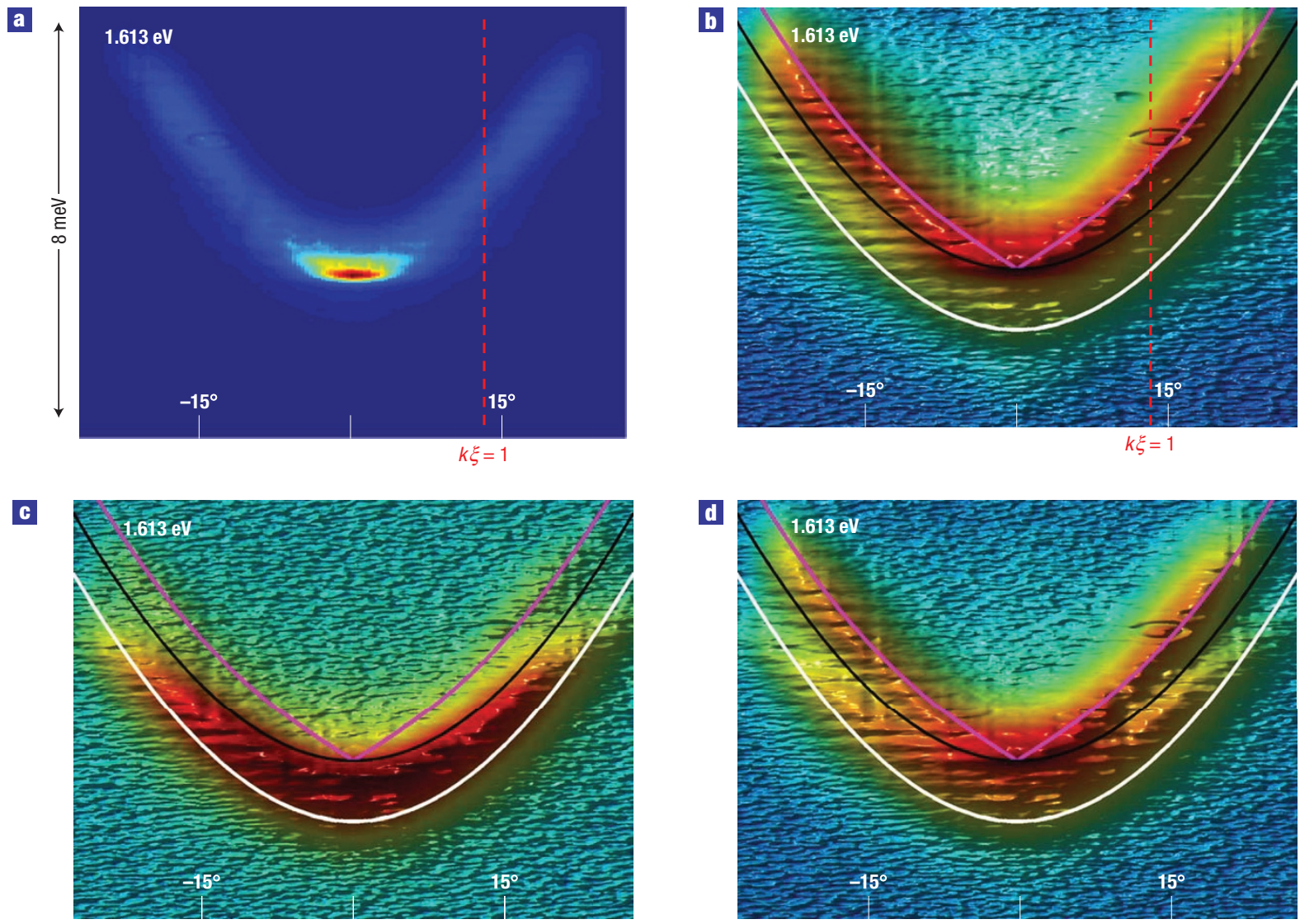

Figure 2 Polarization dependency of the excitation spectrum for an untrapped condensate system. a, A linear plot of the intensity; $\mathbf{b}$ - $\mathbf{d}$ three-dimensional logarithmic plots of the intensity to magnify the excitation spectra. Time-integrated dispersion relation between the LP energy (in the range of 8 meV centred at $1.609 \mathrm{eV}$ ) versus in-plane wavenumber for the untrapped condensate system, where the detuning parameter is $\Delta=1.4 \mathrm{meV}$ and the pump rate is $P=3 P_{\text {th }}\left(P_{\text {th }}=17 \mathrm{~mW}\right)$. A circularly polarized pump beam was incident with an angle of $60^{\circ}$. Three detection schemes: detection of the leakage photons with the cocircular polarization as the pump beam (a,b), detection of the cross-circular polarization (c) and detection of a small amount of mixture of the cocircular polarization with the cross-circular polarization (d). The theoretical curves represent the Bogoliubov excitation energy $E_{\mathrm{B}}$ (pink line), the quadratic dispersion relations $E_{\mathrm{LP}}$ (black line), which start from the condensate energy, and the non-interacting free-polariton dispersion relation $E_{\mathrm{LP}}$ (white line), which is experimentally determined by the data taken far below the threshold $P=0.001 P_{\text {th }}$.

the experimental results (blue diamonds). We note that the above-mentioned nonlinear model based on weakly interacting bosons ${ }^{19-21}$ can reproduce the experimental data only in low-polariton-density regimes. Therefore, we use the experimental values (not theoretical values) for $U(n)$ as the interaction energy in subsequent discussions for the universal feature of the Bogoliubov excitations.

The dispersion relations between the LP energy $E$ and in-plane wavenumber $k$ obtained by the angle-resolved spectroscopy are shown for the pump rate above threshold $P / P_{\text {th }}=3$ in Fig. 2. Figure $2 \mathrm{a}$ represents a linear plot of the intensity, whereas Fig. $2 \mathrm{~b}-\mathrm{d}$ use logarithmic plots of the intensity to magnify the excitation spectra. Here we show an 'untrapped' case, where the pump spot size (diameter $\sim 30 \mu \mathrm{m}$ ) is considerably smaller than the trap size (diameter $\sim 90 \mu \mathrm{m}$ ). In such a case, the LP condensate is formed in an area determined by the pump spot size and the pump rate rather than the trap size owing to the limited lateral diffusion and varying spatial density of the LPs ${ }^{12,18}$. Above threshold, two drastic changes are noticed compared with the standard quadratic dispersion observed far below threshold. One is the blueshift of the $k=0 \mathrm{LP}$ energy and the other is the phonon-like linear dispersion relation in the low-momentum regime $|k \xi|<1$, where $\xi=\hbar / \sqrt{2 m U(n)}$ is the healing length. White and black lines in Fig. $2 \mathrm{~b}$ represent the two quadratic dispersion relations, $E_{\mathrm{LP}}=-U(n)+\left((\hbar k)^{2} / 2 m\right)$ and $E_{\mathrm{LP}}^{\prime}=\left((\hbar k)^{2} / 2 m\right)$, where $m$ is the effective mass of the $k=0$ LP. Here we choose the zero energy as the condensate energy for convenience. Neither of the two theoretical curves can explain the measurement result. A solid pink line in Fig. $2 \mathrm{~b}$ is the Bogoliubov excitation energy, given by ${ }^{22,23}$

$$
E_{\mathrm{B}}=\sqrt{E_{\mathrm{LP}}^{\prime}\left(E_{\mathrm{LP}}^{\prime}+2 U(n)\right)}=U(n) \sqrt{(k \xi)^{2}\left[(k \xi)^{2}+2\right]} .
$$

The measured dispersion relation for the excitation energy versus in-plane wavenumber is in good agreement with the Bogoliubov excitation spectrum without any fitting parameter.

A circularly polarized pump laser beam was used to inject spin-polarized LPs in this experiment. In such a case, the LP condensate preserves the original spin polarization of optically injected polaritons ${ }^{24}$. The result shown in Fig. $2 \mathrm{~b}$ was taken for detecting the leakage photons with the same circular polarization as that of the pump (cocircular detection). If leakage photons with cross-circular polarization were detected, the standard quadratic dispersion was obtained but with slightly blueshifted energy, as shown in Fig. 2c. Even though the circularly polarized pump beam is injected, a small number of cross-circularly polarized photons was detected because of the spin-flip relaxation during 

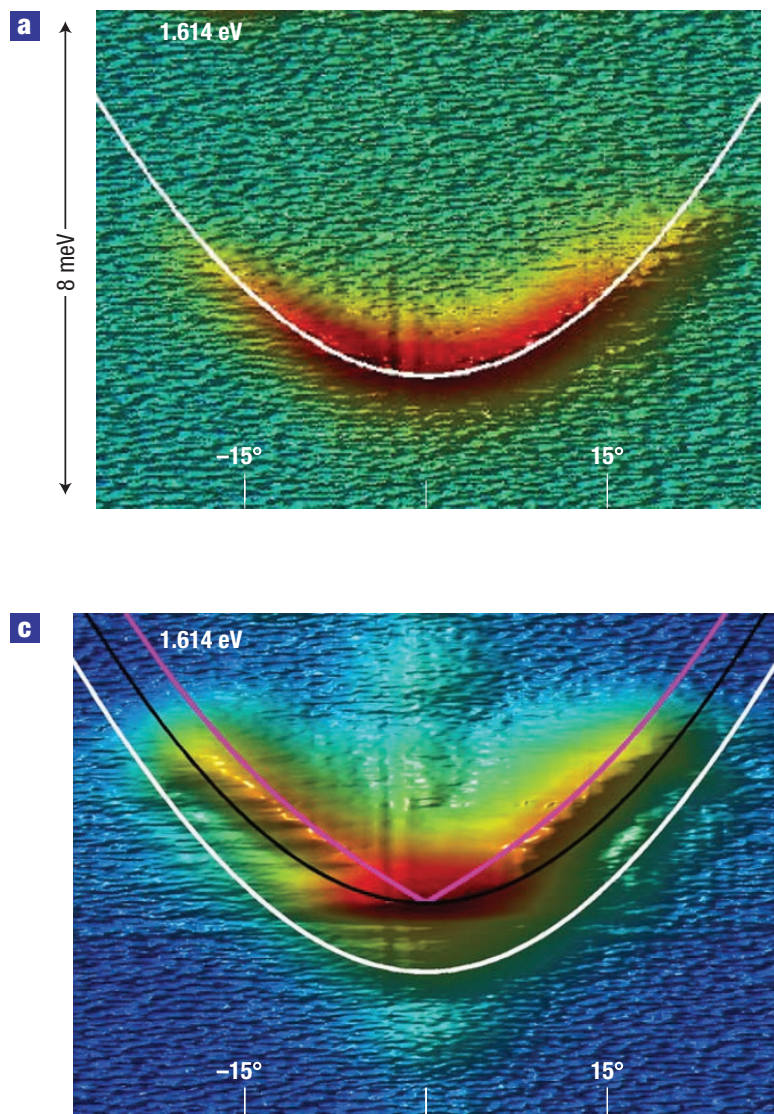
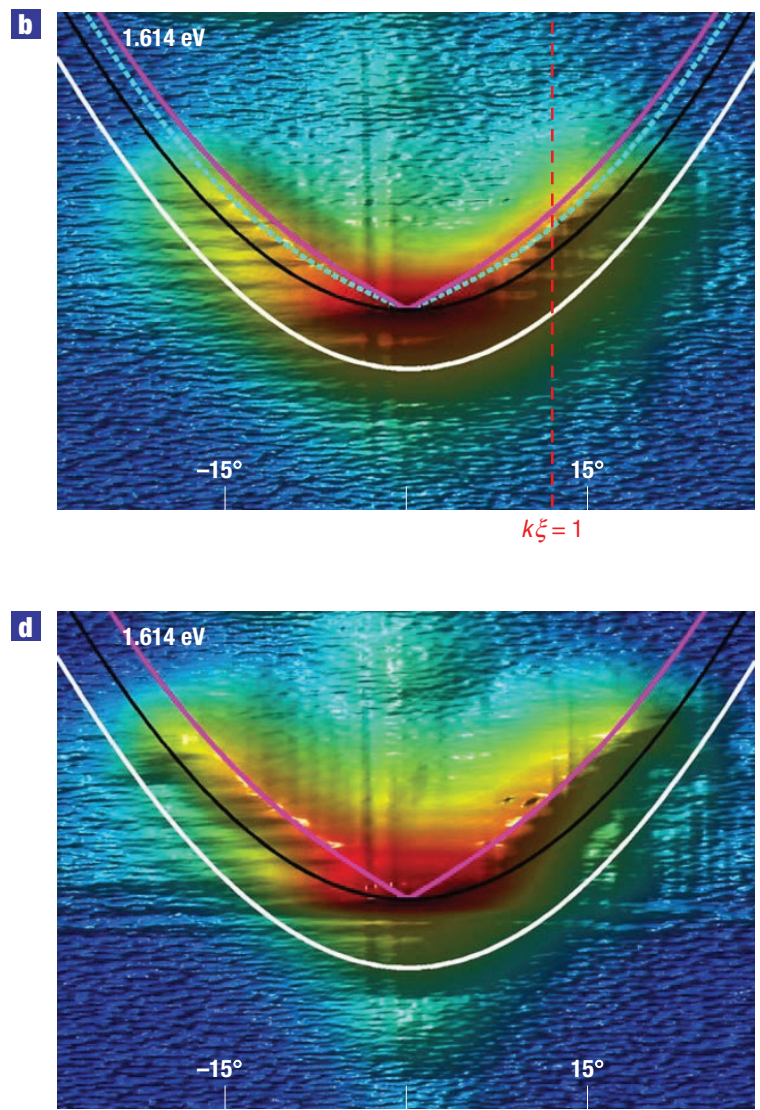

Figure 3 Pump-rate dependency of the excitation spectrum for a trapped condensate system. a-d, Time-integrated dispersion relations between the LP energy (in the range of $8 \mathrm{meV}$ centred at $1.61 \mathrm{eV}$ ) versus in-plane wavenumber. The circularly polarized pump beam is injected into a trap with $8 \mu \mathrm{m}$ diameter, where the detuning parameter is $\Delta=1.6 \mathrm{meV}$. Pump rates are $P=0.05 P_{\text {th }}(\mathbf{a}), P=1.2 P_{\text {th }}(\mathbf{b}), P=4 P_{\text {th }}(\mathbf{c})$ and $P=6 P_{\text {th }}(\mathbf{d})$, where $P_{\text {th }}=4 \mathrm{~mW}$. Three theoretical curves represent the Bogoliubov excitation energy $E_{\mathrm{B}}$ on the basis of the homogeneous model (pink line), the quadratic dispersion curve $E_{\mathrm{LP}}^{\prime}$ starting from the condensate energy (black line) and the non-interacting free-polariton quadratic dispersion curve $E_{\mathrm{LP}}$ (white line) that is determined by the experimental data shown in $\mathbf{a}$. In $\mathbf{b}$ the light-blue dotted line shows the Bogoliubov excitation curve based on the local-density approximation (Supplementary Information, S3).

a cooling process ${ }^{24}$. On increasing the pump rate, the number of LPs with opposite spin is also increased, so their energy is blueshifted compared with the single-polariton energy $E_{\mathrm{LP}}$ (white line) in Fig. 2c. In Fig. 2d we intentionally mixed a small number of the cocircular polarized photons to be detected with the cross-circular polarized photons. The difference between the Bogoliubov excitations with cocircular polarization and the standard quadratic excitation with cross-circular polarization is clearly seen.

The dispersion curves for the LP condensate in a trap with $8 \mu \mathrm{m}$ diameter are shown in Fig. 3 for below and above threshold. The increasing blueshift of the condensate energy with the pump rate is seen from Fig. $3 b$ to $d$. The Bogoliubov excitation energy is modified if the density of a condensate is not homogeneous in space owing to trapping in a finite $\operatorname{size}^{25}$. The light-blue dotted line in Fig. $3 \mathrm{~b}$ is the modified Bogoliubov excitation energy based on the local density-dependent excitation energy $E_{\mathrm{B}}(r)=\sqrt{E_{\mathrm{LP}}^{\prime}\left(E_{\mathrm{LP}}^{\prime}+2 U(n)\right)}$ and the spatial average of $E_{\mathrm{B}}(r)$, where $U(n)$ varies with position (Supplementary Information, S3). In the phonon-like regime $(|k \xi|<1)$, the Bogoliubov dispersion is still linear, $E \approx C^{\prime} k$. However, the speed of sound is not equal to $C=\sqrt{U(n) / m}$ as in the homogeneous case, but is slightly reduced. In the free-particle regime $(|k \xi|>1)$, the dispersion is still quadratic, $E_{\mathrm{B}}(|k \xi|>1) \approx U^{\prime}(n)+\left((\hbar k)^{2} / 2 m\right)$. Here, the off-set energy $U^{\prime}(n)$ is also given by the spatial average of $U(n)$. Just above threshold, the experimental result agrees with such an inhomogeneous model (light-blue dotted line) rather than a homogeneous model (pink solid line). However, as shown in Fig. $3 c$,d, the excitation spectra of the trapped condensates well above threshold are well reproduced by the homogeneous model. According to the local density approximation, at relatively high pump rates, the trapped condensate spreads over the entire trap owing to the repulsive interaction among condensate polaritons and thus interacts with the excitations uniformly. Such a system can be well described by the homogeneous model (Supplementary Information, S3).

As indicated by equation (2), the Bogoliubov excitation energy normalized by the interaction energy $E_{\mathrm{B}} / U(n)$ is a universal function of the wavenumber normalized by the healing length $k \xi$. In Fig. 4a, this universal relation (green solid line) is compared with the experimental results for four different untrapped condensate systems. The experimental data shown in Fig. 4 a are taken by numerical search for the intensity maximum wavenumber for varying $E$ values. In both the phonon-like regime at $|k \xi|<1$ and the free-particle regime at $|k \xi|>1$, the experimental results agree well with the universal curve. On the other hand, at a pump rate far below threshold, the measured dispersion relation is completely described by the single-polariton energy $E_{\mathrm{LP}}$ (red solid line). 
a

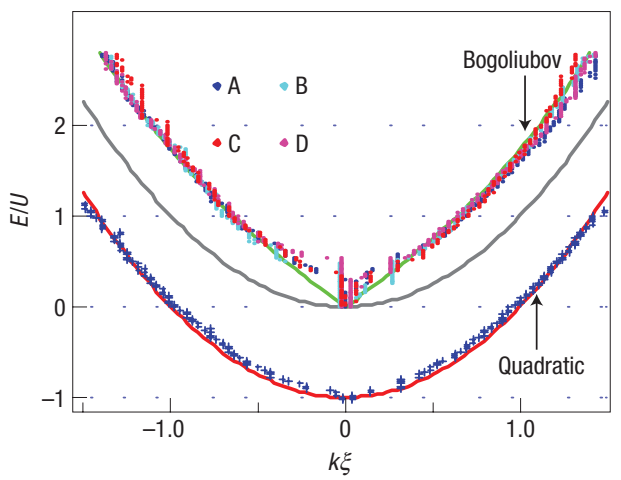

G

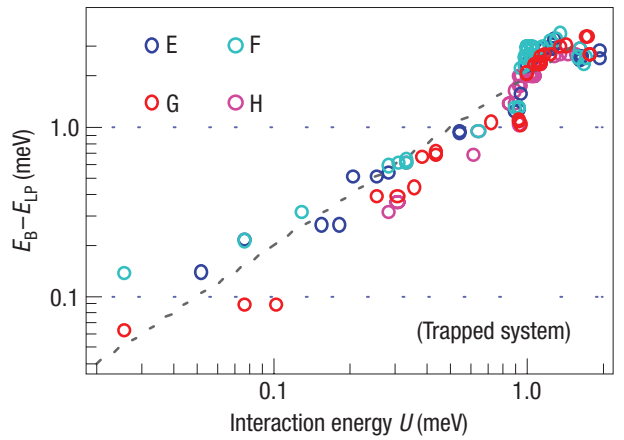

b

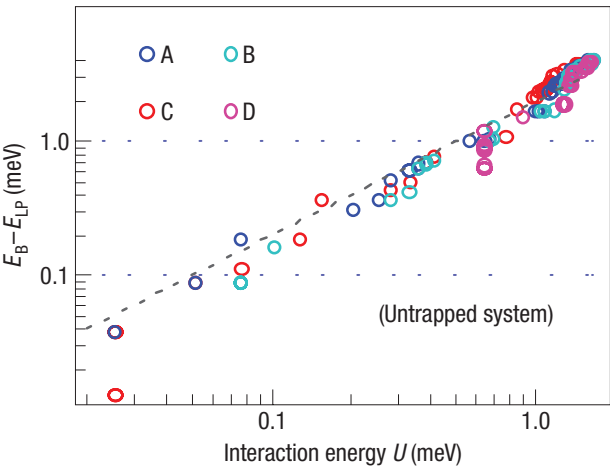

d

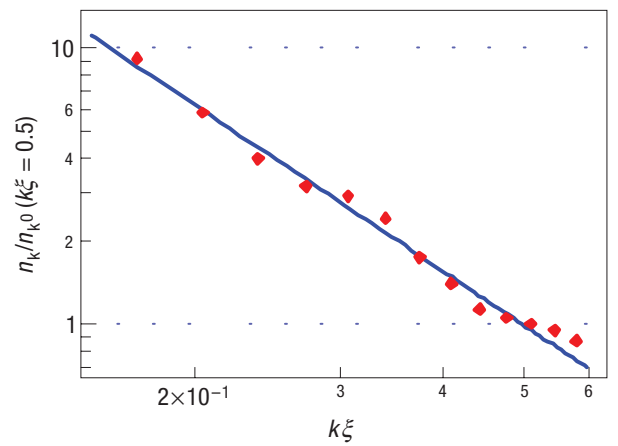

Figure 4 Dispersion relation, energy shift in free-particle regime and population distribution in excitation spectrum. a, Numerically searched excitation energy normalized by the interaction energy $E / U(n)$ as a function of normalized wavenumber $k \xi$ for four different untrapped condensate systems. $\mathrm{A}, \Delta=1.41 \mathrm{meV}, P=4 P_{\text {th }}$ $\left(P_{\text {th }}=6.3 \mathrm{~mW}\right) ; \mathrm{B}, \Delta=0.82 \mathrm{meV}, P=8 P_{\text {th }}\left(P_{\text {th }}=8.2 \mathrm{~mW}\right) ; \mathrm{C}, \Delta=4.2 \mathrm{meV}, P=4 P_{\text {th }}\left(P_{\text {th }}=6.4 \mathrm{~mW}\right) ; \mathrm{D}, \Delta=-0.23 \mathrm{meV}, P=24 P_{\text {th }}\left(P_{\text {th }}=8.2 \mathrm{~mW}\right)$. The experimental data far below threshold are also plotted by blue crosses for system $A$. Three theoretical dispersion curves normalized by the interaction energy are plotted: the Bogoliubov excitation energy $E_{\mathrm{B}} / U(n)$ starting from the condensate energy (green solid line), the quadratic dispersion curves $E_{\mathrm{LP}}^{\prime} / U(n)$ (grey solid line) and the free-polariton dispersion $E_{\mathrm{LP}} / U(n)$ (red solid line). b,c, The energy shift $E_{\mathrm{B}}-E_{\mathrm{LP}}$ in the free-particle regime $(|k \xi|=1)$ is plotted as a function of the interaction energy $U(n)$ for the same four different untrapped systems as in $\mathbf{a}(\mathbf{b})$ and for four different trapped systems (c), where trapped condensate systems are labelled as follows: $\mathrm{E}, d($ diameter $)=7 \mu \mathrm{m}, \Delta=3.3 \mathrm{meV} ; \mathrm{F}$, $d=7 \mu \mathrm{m}, \Delta=2.9 \mathrm{meV} ; \mathrm{G}, d=8 \mu \mathrm{m}, \Delta=1.6 \mathrm{meV} ; \mathrm{H}, d=8 \mu \mathrm{m}, \Delta=2.5 \mathrm{meV}$. The dashed line represents the theoretical prediction on the basis of the homogeneous model $E_{\mathrm{B}}-E_{\mathrm{LP}}=2 U(n)$. d, LP population distribution normalized by the value at $k \xi=0.5$ for the trapped system G (in $\mathbf{c}$ ) at the pump rate $P=2 P_{\mathrm{th}}\left(P_{\mathrm{th}}=4 \mathrm{~mW}\right.$ ). The theoretical $1 / k^{2}$ dependency for the thermal depletion is shown by the blue solid line. The experimental data are plotted in the range of $k \xi=0.2-0.6$ because the experimental data at $|k \xi|<0.2$ are dominated by the condensate with a finite $\Delta k$ and the Bogoliubov excitation is suppressed (see Supplementary Information, S3).

The sound velocity deduced from the phonon-like linear dispersion spectrum is of the order of $\sim 10^{8} \mathrm{~cm} \mathrm{~s}^{-1}$. This value is eight orders of magnitude larger than that of atomic BEC. This enormous difference comes from the fact that the polariton mass is eight orders of magnitude smaller than the atomic mass and the polariton interaction energy is seven orders of magnitude larger than the atomic interaction energy. According to the Landau criterion $^{26}$, the observation of this linear dispersion in the low-momentum regime is an indication of superfluidity in the exciton-polariton system. However, we note that a polariton system is a dynamical system with a finite lifetime, so the Landau criterion might be modified on a quantitative level.

In the free-particle regime $(|k \xi|>1)$, the excitation energy associated with the condensate is larger by $2 U(n)$ than that of a single LP for the same wavenumber. In Fig. 4b,c, this important prediction of the Bogoliubov theory is compared with the experimental results for four different untrapped and trapped condensate systems, respectively. The experimental data were determined as the difference between the measured excitation energy with the presence of the condensate and the standard quadratic dispersion for a single LP state, which is determined by the experimental data obtained for a pump rate far below threshold $\left(P / P_{\text {th }} \ll 1\right)$. The experimental data were taken for varying pump rates in the range of $P / P_{\text {th }} \gg 1$ so that the homogeneous model can be applied to both untrapped and trapped cases. The experimental data are in good agreement with the theoretical curve (grey dashed line) for both untrapped and trapped cases.

Figure $4 \mathrm{~d}$ shows the normalized LP number $n_{k} / n_{k}^{0}$ versus the normalized wavenumber $|k \xi|$ for a trapped condensate, where $n_{k}^{0}$ is evaluated at $|k \xi|=0.5$ for convenience. The LP occupation number $n_{k}$ in the excitation spectrum can be calculated by applying the Bose-Einstein distribution for the Bogoliubov quasiparticles and subsequently taking the inverse Bogoliubov transformation ${ }^{22}$,

$$
n_{k}=\left|v_{-k}\right|^{2}+\frac{\left|u_{k}\right|^{2}+\left|v_{-k}\right|^{2}}{\exp \left(\beta E_{\mathrm{B}}\right)-1},
$$

where $u_{k}, v_{-k}= \pm\left[\frac{(\hbar k)^{2} / 2 m+U(n)}{2 E_{\mathrm{B}}} \pm \frac{1}{2}\right]^{1 / 2}$ and $\beta=\frac{1}{k_{\mathrm{B}} T}$.

The first and second terms of the right-hand side of equation (3) represent the real particles (exciton-polaritons) created by the quantum depletion and the thermal depletion, respectively. In the present polariton condensate system, the thermal depletion is much stronger than the quantum depletion, so the second term of the right-hand side of equation (3) dominates over the first term 
(Supplementary Information, S4). In such a case, the LP population is approximated by $n_{k} \approx\left(m k_{\mathrm{B}} T /(\hbar k)^{2}\right)$ in the small-| $k \xi \mid$ regime, whereas the LP population is given by $n_{k} \approx(1 / 2 \sqrt{2})(1 / k \xi)$ if the quantum depletion is dominant ${ }^{22}$. This theoretical prediction of $1 / k^{2}$ dependency of $n_{k}$ for thermal depletion is compared with the experimental data in Fig. $4 \mathrm{~d}$ and reasonable agreement was obtained.

Received 25 February 2008; accepted 25 June 2008; published 1 August 2008.

\section{References}

1. Einstein, A. Quantentheorie des einatomigen idealen Gases: Zweite Abhandlung. Sitzungber. Preuss. Akad. Wiss. 1, 3-14 (1925).

2. Bogoliubov, N. N. On the theory of superfluidity. J. Phys. USSR 11, 23-32 (1947).

3. Anderson, M. H. et al. Observation of Bose-Einstein condensation in a dilute atomic vapor. Science 269, 198-201 (1995)

4. Stamper-Kurn, D. M. et al. Excitation of phonons in a Bose-Einstein condensate by light scattering. Phys. Rev. Lett. 83, 2876-2879 (1999).

5. Imamoglu, A., Ram, R. J., Pau, S. \& Yamamoto, Y. Nonequilibrium condensates and lasers without inversion: Exciton-polariton lasers. Phys. Rev. A 53, 4250-4253 (1996).

6. Dang, L. S. et al. Stimulation of polariton photoluminescence in semiconductor microcavity. Phys. Rev. Lett. 81, 3920-3923 (1998).

7. Senellart, P. \& Bloch, J. Nonlinear emission of microcavity polaritons in the low density regime. Phys. Rev. Lett. 82, 1233-1236 (1999).

8. Savvidis, P. G. et al. Angle-resonant stimulated polariton amplifier. Phys. Rev. Lett. 84, $1547-1550$ (2000).

9. Deng, H. et al. Condensation of semiconductor microcavity exciton polaritons. Science 298, 199-202 (2002)

10. Kasprzak, J. et al. Bose-Einstein condensation of exciton polaritons. Nature 443, 409-414 (2006).

11. Balili, R. et al. Bose-Einstein condensation of microcavity polaritons in a trap. Science 316, 1007-1010 (2007)

12. Deng, H. et al. Spatial coherence of a polariton condensate. Phys. Rev. Lett. 99, 126403 (2007).

13. Deng, H. et al. Quantum degenerate exciton-polaritons in thermal equilibrium. Phys. Rev. Lett. 97, $146402(2006)$.

14. Sarchi, D. \& Savona, V. Spectrum and thermal fluctuations of a microcavity polariton Bose-Einstein condensate. Phys. Rev. B 77, 045304 (2008).
15. Shelykh, I. A., Malpuech, G. \& Kavokin, A. V. Bogoliubov theory of Bose-condensates of spinor exciton-polaritons. Phys. Status Solidi A 202, 2614-2620 (2005)

16. Keeling, J., Marchetti, F. M., Szymanska, M. H. \& Littlewood, P. B. Collective coherence in planar semiconductor microcavities. Semicond. Sci. Technol. 22, R1-R26 (2007).

17. Wyatt, A. F. G. Evidence for a Bose-Einstein condensate in liquid ${ }^{4} \mathrm{He}$ from quantum evaporation. Nature 391, 56-59 (1997).

18. Lai, C.W. et al. Coherent zero-state and $\pi$-state in an exciton-polariton condensate array. Nature 450 , 529-532 (2007).

19. Ciuti, C. et al. Role of the exchange of carriers in elastic exciton-exciton scattering in quantum wells Phys. Rev. B 58, 7926-7933 (1998).

20. Rochat, G. et al. Excitonic Bloch equations for a two-dimensional system of interacting excitons. Phys. Rev. B 61, 13856-13862 (2000).

21. Schmitt-Rink, S., Chemla, D. S. \& Miller, D. A. B. Theory of transient excitonic optical nonlinearitie in semiconductor quantum-well structures. Phys. Rev. B 32, 6601-6609 (1985).

22. Pitaevskii, L. P. \& Stringari, S. Bose-Einstein Condensation (Clarendon, Oxford, 2003).

23. Ozeri, R., Katz, N., Steinhauer, J. \& Davidson, N. Colloquium: Bulk Bogoliubov excitations in a Bose-Einstein condensate. Rev. Mod. Phys. 77, 187-205 (2005).

24. Deng, H. et al. Polariton lasing versus photon lasing in a semiconductor microcavity. Proc. Natl Acad. Sci. USA 100, 15318-15323 (2003).

25. Stenger, J. et al. Bragg spectroscopy of a Bose-Einstein condensate. Phys. Rev. Lett. 82, 4569-4573 (1999)

26. Landau, L. D. \& Lifshiëtís, E. M. Fluid Mechanics 2nd edn (Pergamon, Oxford, 1987).

Supplementary Information accompanies this paper on www.nature.com/naturephysics.

\section{Acknowledgements}

This work was supported by the JST/SORST programme and Special Coordination Funds for Promoting Science and Technology in Japan. We thank T. Maruyama for support and S. Sasaki for device fabrication.

Author contributions

S.U. carried out the experiments, analysed the data and wrote the paper, L.T. theoretically studied the data, G.R carried out the experiments and analysed the data, C.W.L conceived, designed and carried out the experiments, N.K., T.F., M.G., A.L., S.H. and A.F. prepared materials and experimental tools and Y.Y. conceived the project in this paper.

\section{Author information}

Reprints and permission information is available online at http://npg.nature.com/reprintsandpermissions. Correspondence and requests for materials should be addressed to S.U. or Y.Y. 